\title{
Isolamento e Identificação de Fungos em Fezes de Pombos nas Dependências da Universidade de Gurupi (UNIRG)
}

\author{
Isolation and Identification of Fungi in Powder Fairs in University de Gurupi Dependencies \\ (UNIRG)
}

Débora Zvicker da Silva1, Joelcy Pereira Tavares², Ribamar Aires Cabral junior $^{3}$, Tânia Fernandes Machado Silva ${ }^{4}$. Marcos Gontijo da Silva ${ }^{5}$, Érica Eugênio Lourenço Gontijo6.

\section{RESUMO}

Introdução:As fezes dos pombos domésticos criam ambientes apropriados para o crescimento de fungos ambientais e patogênicos ao homem.Objetivo: Isolar e identificar fungos em fezes de pombos nas dependências da Universidade de Gurupi (UNIRG), estado do Tocantins. Metodologia:Foram coletadas 10 amostras de $5 \mathrm{~g}$ de fezes a cada 2 (dois) meses nos telhados e caixas d'água da Universidade. Foram feitas 6 (seis) coletas, totalizando 60 amostras. As amostras foram inseridas em tubos de ensaio com $5 \mathrm{ml}$ de solução fisiológica, e semeada por espalhamento com swab de algodão estéril em meio de cultura ágar Sabouraud dextrose e incubado em estufa com temperatura a $25^{\circ} \mathrm{C}$ por 30 dias. Foi realizado a visualização e identificação da espécie fúngica em microscópio óptico com objetivas de 10 e 40x. Os dados foram tratados por estatística descritiva. Os resultados dos meses secos foram comparados com os dos meses chuvosos por meio do teste de Odds Ratio com $p \leq 5 \%$.Resultados: Em 78.33\% foi possível isolar fungos. Os fungos mais freqüentes foram: Aspergillusspp em $34.04 \%$, Fusariumspp em 28\%, Peniciliumspp em $12.77 \%$ e Candidasppem $10.64 \%$ das amostras positivas. Conclusão: Foi encontrada alta freqüência de fezes contaminadas por fungos e algumas.

Descritores: Fungos ambientais, fezes de pombo, infecções laborais.

\section{ABSTRACT}

Introduction: The faeces of domestic pigeons create environments suitable for the growth of environmental fungi and pathogenic to humans. Objective: To isolate and identify fungi in feces of pigeons at the University of Gurupi (UNIRG), state of Tocantins. Methodology: Ten samples of $5 \mathrm{~g}$ of feces were collected every 2 (two) months on the roofs and water tanks of the University. Six (6) collections were made, totaling 60 samples. The samples were placed in test tubes with $5 \mathrm{ml}$ of saline solution and seeded by spreading with sterile cotton swab in Sabouraud agar dextrose medium and incubated in an oven with a temperature of $25^{\circ} \mathrm{C}$ for 30 days. Visualization and identification of the fungal species were carried out under an optical microscope with 10 and $40 x$ objective. The data were treated by descriptive statistics. The results of the dry months were compared with those of the rainy months by means of the Odds Ratio test with $p \leq 5 \%$. Results: In $78.33 \%$ it was possible to isolate fungi. The most frequent fungi were: Aspergillusspp in $34.04 \%$, Fusariumspp in $28 \%$, Peniciliumspp in $12.77 \%$ and Candidaspp in $10.64 \%$ of the positive samples. Conclusion: There was a high frequency of feces of fungi contaminated by fungi and some species found have a serious pathogenic potential for man.

Descriptors: Medication Advertisement. Influence of Propaganda. Self-medication.

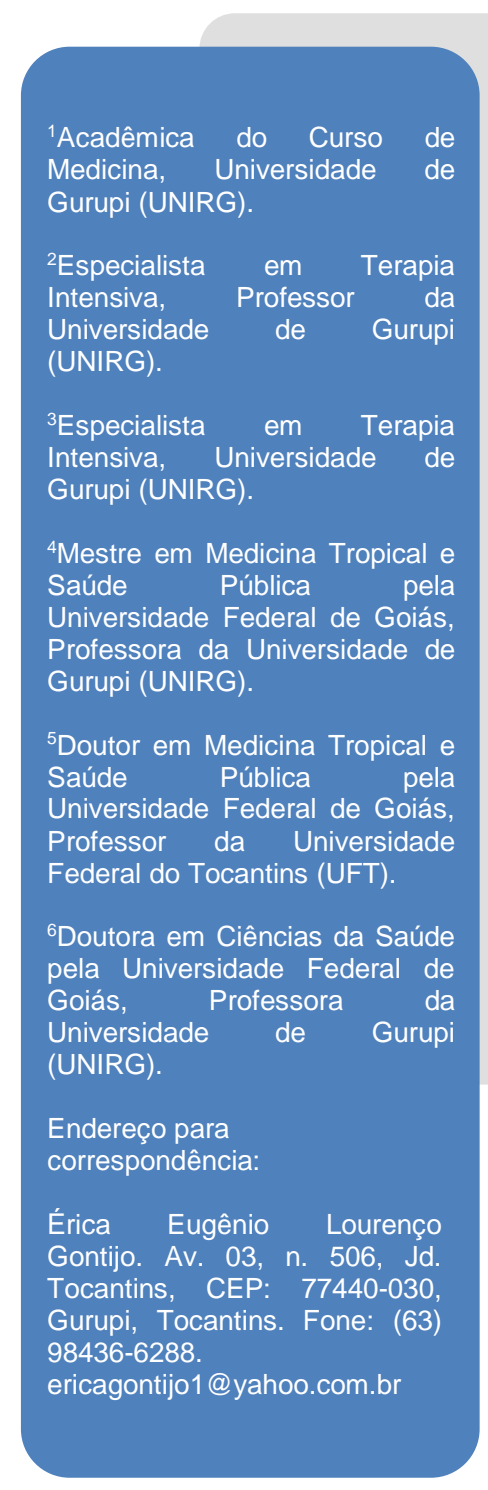
Medicina, Universidade de Gurupi (UNIRG).

Intensiva,

Universidade

em

rapia

(UNIRG).

${ }^{3}$ Especialista em Terapia

Intensiva, Universidade de Gurupi (UNIRG).

${ }^{4}$ Mestre em Medicina Tropical e Saúde Pública pela Universidade Federal de Goiás, Professora da Universidade de Gurupi (UNIRG).

${ }^{5}$ Doutor em Medicina Tropical e Saúde Pública pela Universidade Federal de Goiás, Professor da Universidade Federal do Tocantins (UFT).

${ }^{6}$ Doutora em Ciências da Saúde pela Universidade Federal de Goiás, Professora da Universidade (UNIRG).

Endereço para

correspondência:

Érica Eugênio Lourenço

Gontijo. Av. 03, n. 506, Jd.

Tocantins, CEP: 77440-030

Gurupi, Tocantins. Fone: (63)

98436-6288.

ericagontijo1@yahoo.com.br 


\section{INTRODUÇAO}

Os pombos domésticos Columba livia pertencem à Família Columbidae, Ordem Columbiformes, encontram-se distribuídos mundialmente sendo difícil de determinar pela sua longa história de domesticação. No Brasil, podem ser encontrados nas áreas rurais e urbanas, pela facilidade de encontrarem alimento e água abundantes, além de sítios localizados em áreas altas e protegidas, edifícios, estátuas, vigas de telhados, parapeitos de janelas, galpões e obras inacabada como área de pouso, abrigo e nidificação ${ }^{1,2}$.

Com isso, muitos são os problemas encontrados pelo acúmulo de fezes, penas e restos de ninhos, levando a entupimentos de sistemas de drenagem de águas pluviais, comprometendo o funcionamento de equipamentos diversos e favorecendo maiores riscos de contaminações em fontes de água e alimentos. Dentre todos os problemas supracitados destacam-se as doenças que podem ser transmitidas aos seres humanos, principalmente pela grande quantidade de microrganismos patogênicos e parasitas veiculados por essas aves, especialmente em seus excrementos ${ }^{3}$.

Salienta-se que as fezes dos pombos domésticos podem albergar os fungos Aspergillus fumigatus e Histoplasma capsulatum, agentes causadores da histoplasmose, doença que atinge primariamente o sistema respiratório e que também afeta, em particular, os portadores de AIDS. Outras leveduras também são achados freqüentes nas fezes dos pombos domésticos, principalmente as dos gêneros: Candida,Trichosporon, Rhodotorula, Hansenula e Cryptococcus. Esse último gênero destaca-se por ser causador da criptococose, que se caracteriza por infecções pulmonares e infecção grave das meninges, em especial no hospedeiro imunocomprometido ${ }^{2,4}$.

As fezes dos pombos, hoje considerados como domésticos por dividirem de nosso habitat, devido ao seu pH ideal para o crescimento de fungos como o Aspergillus fumigatus, Histoplasma capsulatum, Candida,Trichosporon, Rhodotorula, Hansenula e Cryptococcus são potenciais patogênicos para a saúde humana 5 .

Desse modo, o trabalho se justifica pelo supracitado risco de contaminação que pode representar as fezes contaminadas de pombos e sua concentração na Universidade de Gurupi (UnirG), o que torna esse risco conseqüentemente presente no cotidiano de todos aqueles que fazem uso de tal instalação e pela carência de dados sobre essa forma de disseminação e contágio das doenças fúngicas. 
Sabendo que os excrementos dos pombos domésticos podem ser vetores para doenças graves, inclusive fatais, o objetivo deste estudo é isolar e identificar fungos em fezes de pombos nas dependências da Universidade de Gurupi, estado do Tocantins.

\section{MATERIAIS E METODOS}

A pesquisa foi realizada nas dependências da Universidade de Gurupi (UNIRG), nos blocos A, B e C do Campus II. A UNIRG está localizada na cidade de Gurupi, localizada no Sul do Estado do Tocantins, Brasil. Este Município é a terceira maior cidade do estado, com uma população estimada de 85.523 habitantes, segundo o IBGE ${ }^{6}$.

Foram coletadas amostras de fezes de pombos dos locais mais infestados por pombos nos blocos A, B e C da IES onde estão sediadas salas de aulas teóricas, laboratórios de aulas práticas, coordenações de curso e biblioteca. No campus II da IES estão em funcionamento os cursos de Medicina, Enfermagem, Fisioterapia, Farmácia, Educação Física, Psicologia e Ciências Contábeis com circulação aproximada de 3 (três) mil pessoas por dia.

Por se tratar de uma pesquisa de campo para análise de fezes de pombos e, portanto, não envolver seres humanos nem manipulação de animais, o trabalho não precisou de aprovação do Comitê de Ética em Pesquisa Humano ou Animal.

De forma bimestral foram realizadas 10 (dez) coletas de amostras fecais de pombos em locais diversos como telhados e caixas d'água onde era possível a visualização de pombos.

O clima do estado do Tocantins é predominante o tropical seco, que é caracterizado por uma estação chuvosa (de outubro a abril) e outra seca (de maio a setembro). Foram realizadas 6 (seis coletas), sendo 3 (três) em período chuvoso e 3(três) em período de estiagem (tempo seco). Cada coleta foi formada por 10 (dez) amostras de diferentes locais, no total o trabalho foi constituído por (60) sessenta amostras.

Foram coletados aproximadamente $5 \mathrm{~g}$ de fezes com o auxílio de uma pinça estéril. As amostras foram inseridas dentro de tubos de ensaio com $5 \mathrm{ml}$ de água destilada, esterilizados, os mesmos foram fechados e identificados e encaminhadas ao laboratório de microbiologia da Universidade de Gurupi. Lá por meio de swabde algodão estéril o material foi semeado por espalhamento em meio de cultura ágar sabourauddextrosee incubado em estufa com temperatura a $25^{\circ} \mathrm{C}$ por 30 dias. 
Para se obter um tapete uniforme de crescimento microbiano, ou colônias isoladas (após diluição) utilizou-se o método de "espalhamento" em placa. Consistiu em espalhar o material (suspensão de fezes) com o auxílio de um swab (para obtenção de tapete uniforme), para obtenção de colônias isoladas após diluição, fazendo a semeadura por toda a superfície da placa de Petri a ser utilizada nesta técnica. Tomou-se o cuidado para que toda a superfície da placa fosse semeada, evitando regiões sem semeadura. $O$ procedimento de semeadura com o swab foi feito em três direções distintas, rodando a placa.

Foi realizado o monitoramento semanal do crescimento de colônias fúngicas que foram identificadas de forma macroscópica e microscópica por meio do corante lactofenol azul de algodão e visualização e identificação da espécie fúngica em microscópio óptico com objetivas de 10 e 40x (Figura 1).

Figura 1: Esquema ilustrativo dos experimentos de isolamento e identificação fúngica em fezes de pombos obtidas nas dependências da Universidade de Gurupi, Tocantins, Brasil, 2018.

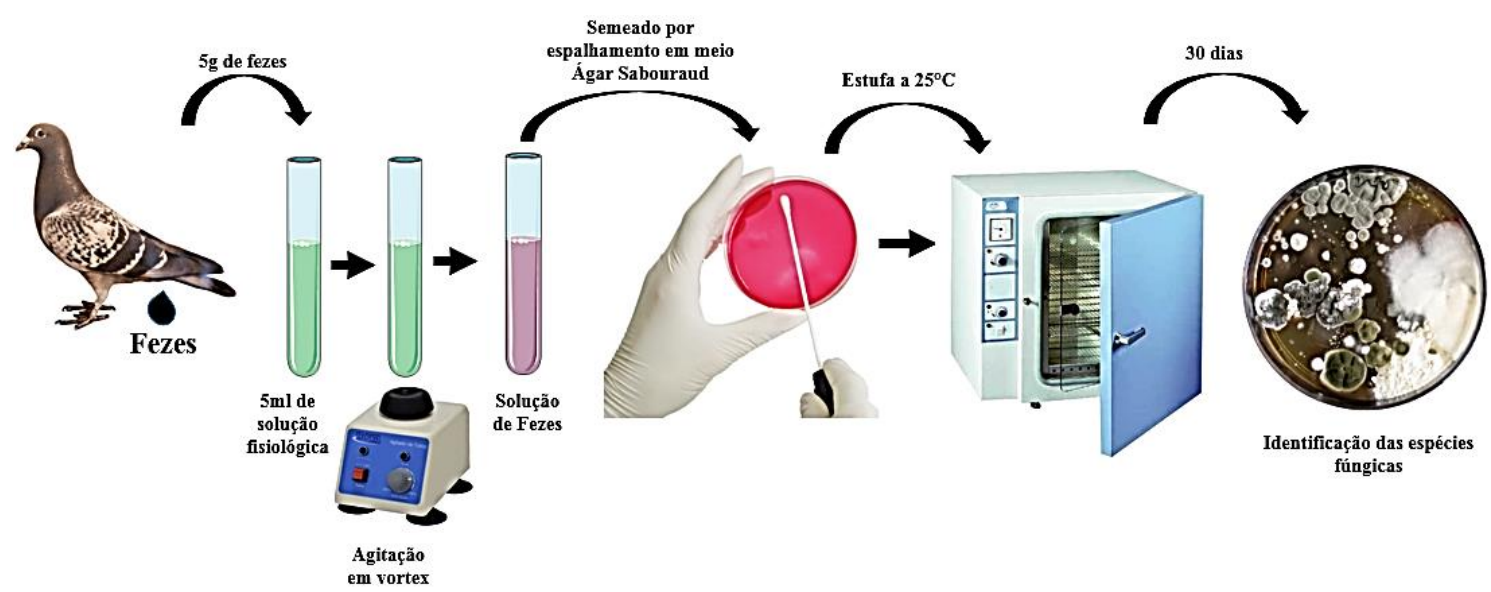

Os dados foram inseridos em planilhas do Excel 2016 e tratados por meio de estatística descritiva, expressos em porcentagem e em medidas de tendência central. Os resultados dos meses secos foram comparados com os dos meses chuvosos por meio do teste de qui-quadrado com $\mathrm{p} \leq 5 \%$

\section{RESULTADOS E DISCUSSĀO}

Das 60 amostras coletadas, em 47 (78.33\%) foi possível isolar e identificar alguma espécie de fungo. Quanto a cronologia das amostras, foi observado que das 30 amostras coletadas no período chuvoso 25 (83.33\%) e das 30 amostras coletadas no 
período de estiagem 22 (73.33\%) estavam positivos. Não foi observado diferença significativa na taxa de contaminação fúngica nos diferentes períodos do ano OR: 1.818; IC: 0.52-6.38; p: 0.53 (Figura 2).

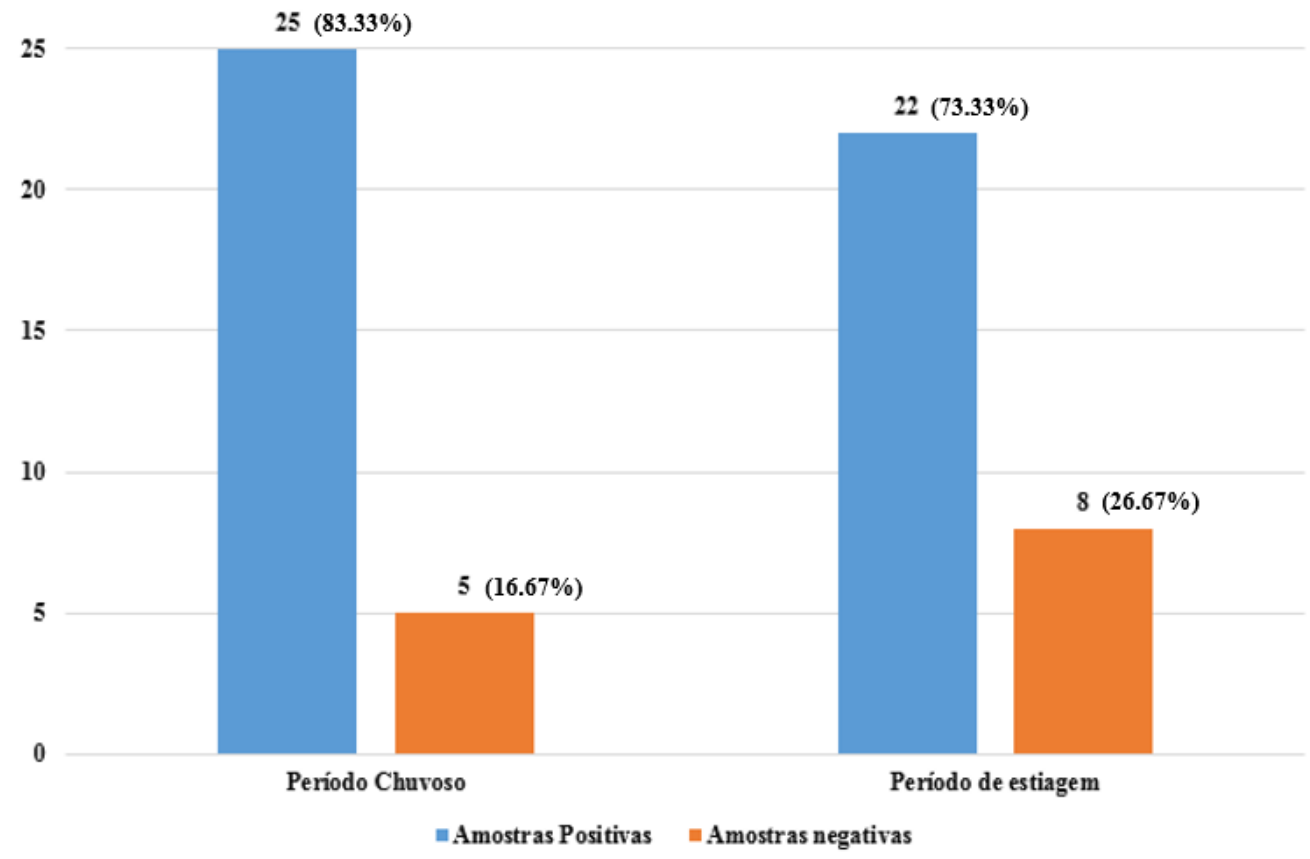

Figura 2: Frequência de contaminação fúngica em fezes de pombos obtidas nas dependências da Universidade de Gurupi, Tocantins, Brasil, 2018 em período chuvoso e de estiagem.

A grande quantidade de pombos encontradas nas dependências da Universidade de Gurupi, é semelhante à de outras regiões urbanas do país. Estes, apresentam grande facilidade de adaptação nos centros urbanos, favorecida pela ausência de predadores naturais, abundância de alimentos e locais para habitação, e, apesar de poder voar, é um animal que vive entre os pedestres das cidades: seu plano de atuação se dá no mesmo plano que o dos habitantes humanos, de forma que é um dos animais mais visíveis e presentes no cotidiano ${ }^{3,7}$.

Grandes concentrações de pombos em determinada área trazem complicações não só como problema estético e sujidade no local, mas como um risco potencial à saúde pública $^{8}$. Para a literatura, as principais zoonoses transmitidas pelos pombos são a criptococose, a clamidiose, a salmonelose e a histoplasmose ${ }^{9}$, sendo também responsáveis pela transmissão ornitose, toxoplasmose, encefalite, dermatites, alergias respiratórias, meningite, psitacocose e aspergilose. A via de transmissão mais comum destas zoonoses são as fezes ressecadas em suspensão, que podem ser inaladas ${ }^{2,10}$. 
DOI 10.18606/2318-1419/amazonia.sci.health.v7n2p79-86 Revista Amazônia: Science \& Health
Silva DZ, Tavares JP, Junior RAC, Silva TFM, Silva MG, Gontijo EEL

Isolamento e Identificação de Fungos em Fezes de Pombos nas Dependências da Universidade de Gurupi (UNIRG)

Quanto aos microrganismos mais freqüentes se destacaram a presença de fungos do gênero Aspergillussppque representaram $34.04 \%$ dos fungos isolado, Fusariumspp que representou $28 \%$ das amostras positivas, Peniciliumsppcom $12.77 \%$ das amostras positivas e Candidasppcom 10.64\% (Tabela 1).

Tabela 1: Espécies fúngicas em fezes de pombos obtidas nas dependências da Universidade de Gurupi, Tocantins, Brasil, 2018.

\begin{tabular}{|c|c|c|c|c|c|c|}
\hline & \multicolumn{3}{|c|}{ Meses Chuvosos } & \multicolumn{3}{|c|}{ Meses de estiagem } \\
\hline & Coleta 1 & Coleta 2 & Coleta 3 & Coleta 4 & Coleta 5 & Coleta 6 \\
\hline Amostra 1 & $\begin{array}{l}\text { Aspergillus } \\
\text { niger }\end{array}$ & $\begin{array}{c}\text { Aspergillus } \\
\text { fumigatus } \\
\text { Aspergillus } \\
\text { flavus } \\
\end{array}$ & Fusariumspp & Candidaspp & Negativo & Negativo \\
\hline Amostra 2 & Negativo & $\begin{array}{l}\text { Aspergillus } \\
\text { fumigatus }\end{array}$ & $\begin{array}{l}\text { Aureobasidium } \\
\text { pullulans }\end{array}$ & Aspergillus flavus & Candidaspp & Negativo \\
\hline Amostra 3 & $\begin{array}{l}\text { Aspergillus } \\
\text { terreus }\end{array}$ & $\begin{array}{c}\text { Fusarium; } \\
\text { Aspergillus niger }\end{array}$ & Negativo & Aspergillus niger & Negativo & $\begin{array}{l}\text { Penicilium } \\
\text { vanoraogei }\end{array}$ \\
\hline Amostra 4 & $\begin{array}{c}\text { Aspergillus } \\
\text { terreus }\end{array}$ & Candida albicans & Fusariumspp & Fusariumspp & Candidaspp & $\begin{array}{l}\text { Scedosporium } \\
\text { apoiospermum }\end{array}$ \\
\hline Amostra 5 & $\begin{array}{l}\text { Penicilium } \\
\text { vanoraogei }\end{array}$ & $\begin{array}{c}\text { Chytriomyces } \\
\text { hialinus }\end{array}$ & Negativo & $\begin{array}{l}\text { Lichtheimia } \\
\text { corimbifera }\end{array}$ & Negativo & Candidaspp \\
\hline Amostra 6 & Negativo & Negativo & $\begin{array}{c}\text { Candida spp; } \\
\text { Fusariumspp; } \\
\text { Aspergillus Niger }\end{array}$ & Aspergillus niger & $\begin{array}{l}\text { Lichtheimia } \\
\text { corimbifera }\end{array}$ & Negativo \\
\hline Amostra 7 & $\begin{array}{l}\text { Penicilium } \\
\text { vanoraogei }\end{array}$ & Fusariumspp & $\begin{array}{l}\text { Lichtheimia } \\
\text { corimbífera }\end{array}$ & Fusariumspp & Negativo & $\begin{array}{l}\text { Aspergillus } \\
\text { fumigatus }\end{array}$ \\
\hline Amostra 8 & $\begin{array}{l}\text { Penicilium } \\
\text { vanoraogei }\end{array}$ & Fusariumspp & Fusariumspp & $\begin{array}{l}\text { Aureobasidium } \\
\text { pullulans }\end{array}$ & Negativo & $\begin{array}{l}\text { Penicilium } \\
\text { vanoraogei }\end{array}$ \\
\hline Amostra 9 & $\begin{array}{l}\text { Aspergillus } \\
\text { alliaceus }\end{array}$ & Fusariumspp & Fusariumspp & $\begin{array}{l}\text { Aspergillus } \\
\text { alliaceus }\end{array}$ & Aspergillus niger & $\begin{array}{l}\text { Penicilium } \\
\text { frequentans }\end{array}$ \\
\hline Amostra 10 & $\begin{array}{c}\text { Aspergillus } \\
\text { terreus }\end{array}$ & Rhizopusspp & Negativo & $\begin{array}{l}\text { Aspergillus } \\
\text { terreus }\end{array}$ & Candidaspp & fladosporiumspp \\
\hline
\end{tabular}

Os resultados deste trabalho se assemelharam quanto a freqüência de fungos do gênero Aspergillusspp e Candidaspp, porem divergiram quanto as demais espécies. Em outros trabalhos se destacam a presença principalmente de Aspergilus fumigatus, Histoplasma capsulatum, causadores da histoplasmose, além das leveduras dos gêneros Candida, Rhodotorula, Trichosporon, Hansenula e Cryptococcus. Todos podem ser potencialmente patogênicos, principalmente naqueles indivíduos imunodeprimidos. Este último gênero caracteriza-se como o causador da criptococose, podendo causar infecções graves das vias respiratórias e das meninges, sendo muito deletério e podendo levar a morte porem não foi encontrado no trabalho ${ }^{1,9}$. 
Os fungos do gênero Aspergillusspp, cerca de 16 a 20 espécies podem infectar o homem causando várias manifestações clínicas, tais como: micetomas, aspergilose alérgica, aspergilose pulmonar e formas graves invasivas generalizadas que podem ser fatal ${ }^{11}$.

Fungos do gênero Fusariumspp infectam plantas e animais, até o homem, sendo responsável por infecções oportunistas que causam lesão na pele, unhas, olhos ou intraperitoneal (peritonite) $)^{11,12}$.

Os fungos do gênero Candidaspp são responsáveis por causar candidíase, doença fúngica mais prevalente no mundo e responsável por grande quantidade de óbitos, porem são mais freqüentemente relatados como oportunistas e causadores de lesões genitais, orais. Podem também causar infecções hospitalares em cateteres de pacienteshospitalizados ${ }^{1,2,5,11,13}$.

No ambiente, C. neoformans é encontrado associado à excretas de pombos e em ocos de árvores. Reolon et al. (2004) ${ }^{14}$ analisaram 88 amostras de excretas de pombos em cinco praças de Porto Alegre, Rio Grande do Sul. Os resultados mostraram que todas as amostras foram positivas para $C$. neoformans nos cinco ambientes estudados pelos autores. Kobayashi et al. (2005) ${ }^{12}$ coletaram um total de 117 amostras de excretas de pombos e outras aves para estudar possíveis fontes ambientais de Cryptococcus spp. na cidade de Goiânia, Goiás; encontrando ocorrência de C. neoformans var. grubii em 36 dessas amostras $(20,3 \%)^{15}$.

Apesar de menos freqüente, outros tipos de fungos também têm sido detectados relacionados a essas aves, destacando-se a Candida albicans, que apesar de perfazer a microbiota normal de um indivíduo saudável, possui potencial patológico de causar quadros de candidíase, principalmente em indivíduos imunocomprometidos ${ }^{13}$.

\section{CONSIDERAÇOES FINAIS}

Pode-se verificar que o pombo doméstico (Columbia livia), pode representar importante fonte carreadora de fungos patogênicos ao homem; assim, este trabalho além de incrementar os estudos ainda escassos sobre o tema na região, mas como forma de voltar-se para a melhoria da atenção preventiva e promoção à saúde laboral de acadêmicos, servidores que circulam as dependências da IES ou que habitam em seu entorno. 
DOI 10.18606/2318-1419/amazonia.sci.health.v7n2p79-86 Revista Amazônia: Science \& Health

2019, Vol. 7, № 2
Silva DZ, Tavares JP, Junior RAC, Silva TFM, Silva MG, Gontijo

Isolamento e Identificação de Fungos em Fezes de Pombos nas Dependências da Universidade de Gurupi (UNIRG)

\section{REFERENCIAS}

1. MARTINS RDA. A origem dos pombos domésticos na estratégia argumentativa de Charles Darwin. Filos. e História da Biol. 2012;7(1):91-116.

2. RIBEIRO MFP, SILVA AM, FERNANDES WS, MELLO MM. Isolamento de Cryptococcus neoformans em fezes de pombos (Columba livia) em praças públicas de São José dos Campos-SPP J Heal. Sci Inst [Internet]. 2017 [cited 2018 Dec 17];35(1):23-30. Available from: https://www3.unip.br/presencial/comunicacao/publicacoes/ics/edicoes/2017/01_janmar/V35_n1_2017_23a27.pdf

3. LIMA CT, KLAFKE GB, XAVIER MO. Cryptococcus spp. em excretas de Columba livia (pombos domésticos) provenientes de um hospital universitário no Sul do Brasil. Arq. Inst. Biol., São Paulo [Internet]. 2015 [cited 2018 Dec 17];82:1-4. Available from: http://www.scielo.br/pdf/aib/v82/18081657-aib-001072013.pdf

4. CONTIN JT, QUARESMA GS, SILVA EF, LINARDI VR. Ocorrência de Cryptococcus neoformans em fezes de pombos na cidade de Caratinga, MG - Brasil. Rev Med Minas Gerais. 2011;21(1):19-24.

5. MENEZES T; SCAIN, G; QUADROS, R. M; MILETTI, L.C; SOUZA, A. L; MIGUEL, R. L; MARQUES SMT. Cryptococcus spp. EM EXCRETAS DE POMBOS ( Columba livia ) DE ÁREAS PÚBLICAS DE LAGES, SANTA CATARINA. Sci. Anim. Heal. 2014;2(2):102-114.

6. IBGE. Estimativas da População dos Municípios e Unidades da Federação Brasileiros com data de referência em $1^{\circ}$ de julho de 2016. Brasília. Retrieved from http://biblioteca.ibge.gov.br/visualizacao/livros/liv97868.pdf. 2016;

7. MUSEU DE CIÊNCIAS NATURAIS, FUNDAÇÃO ZOOBOTÂNICA DO RIO GRANDE DO SUL. POMBOS-DOMÉSTICOS, Sugestões para o controle em escolas públicas estaduais de Porto Alegre [Internet]. Porto Alegre : 2007 [cited 2018 Dec 17]. Available from: http://www.fzb.rs.gov.br/upload/20150514114242pombos_domesticos.pdf

8. Sociedade Brasileira de Infectologia. PARECER TÉCNICO SOBRE DOENÇAS FÚNGICAS TRANSMITIDAS POR AVES [Internet]. 2018 [cited 2018 Dec 17];7. Available from: https://www.infectologia.org.br/admin/zcloud/125/2018/07/c8959e020ef3534d4926a5a9a6cedc75489 2bef43c8af01ad09e6aac3bd8f2c7.pdf

9. RIBEIRO MFP, SILVA AM, FERNANDES WS, MELLO MM. Prevalência de Cryptococcus neoformans nos pombos urbanos da cidade de Porto Alegre, Rio Grande do Sul. J. Bras. Petol.Med. Lab [Internet]. 2004 [cited 2018 Dec 17];40(5):293-298. Available from: https://www.lume.ufrgs.br/bitstream/handle/10183/52926/000443396.pdf?sequence $=1$

10. AGUIAR MB, LUCIANO L. Avaliação dos riscos de contaminação relacionados com a superpopulação de Columbia livia (pombos) em trabalhadores portuários avulsos. Rev. Bras. Pesqui. em Saúde. 2011;13(3):43-49.

11. MARQUES A, PELLI A, MOURA RS, OLIVEIRA ACS, MARSON JM, SILVEIRA LAM, et al. Avaliação da microbiota associada à Pseudolynchia canariensis coletadas em pombos domésticos (Columba livia). Rev. Ciências Médicas e Biológicas [Internet]. 2010 [cited 2018 Dec 17];9(3):224228. Available from: https://repositorio.ufba.br/ri/bitstream/ri/22770/1/8_v.9_3.pdf

12. KOBAYASHI CCBA, SOUZA LKH, FERNANDES OFL. Characterization of Cryptococcus neoformans isolated from urban environmental sources in Goiânia, Goiás State, Brazil. Rev. do Inst. Med. Trop. 2005;47(4):203-207.

13. NUCCI M, COLOMBO A. Candidemia due to Candida tropicalis: clinical, epidemiologic, and microbiologic characteristics of 188 episodes occurring in tertiary care hospitals. Diagn. Microbiol. Infect. Dis. 2007;58:77-82.

14. REOLON A, PEREZ LR., MEZZARI A. Prevalência de Cryptococcus neoformans nos pombos urbanos da Cidade de Porto Alegre, Rio Grande do Sul. J. Bras. Patol. e Med. Lab. 2004;40(5):293298.

15. SEVERO CB, GAZZONI AF, SEVERO LC. Curso de Atualização - Micoses: Capítulo 3 Criptococose pulmonar. J. Bras. Pneumol. 2009;35(11):1136-1144. 